Methods 11 patients with stable angina were prospectively recruited for a third generation dual-source CTCA (prospective ECG-triggered sequential scans, gantry rotation $250 \mathrm{~ms}$, $128 \times 2 \times 0.5 \mathrm{~mm}$ collimation with tube voltage $(\mathrm{kV})$ and current determined by scanner) and 3-vessel intravascular imaging (IVUS). The IVUS pullbacks were manually segmented and plaques with $\mathrm{PB}>40 \%$ were included in the study. Semiautomated CTCA segmentation of lumen and vessel wall borders were performed and corresponding plaques with IVUS were identified. The PB at the minimum lumen area in CTCA and IVUS were estimated and compared.

Results The mean age of the studied population was $61 \pm 11$, $3(27.2 \%)$ were diabetic and $7(63.6 \%)$ suffered from hypercholesterolaemia. Group $1(<100 \mathrm{kV})$ included 6 patients $(16$ plaques) while group $2(\geq 100 \mathrm{kV})$ included 5 patients (11 plaques). CTCA-derived PB was similar between both groups

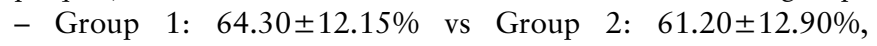
$\mathrm{p}=0.551$. However, Group 2 showed better correlation between IVUS and CTCA-derived PB $(68.00 \pm 13.58 \%$ vs $61.20 \pm 12.90 \%, \mathrm{r}=0.863, \mathrm{p}=0.006)$ compared to Group 1 $(59.79 \pm 9.66 \% \quad$ vs $\quad 64.30 \pm 12.90 \%, \quad \mathrm{r}=0.222, \quad \mathrm{p}=0.427)$, respectively.

Conclusion Higher tube voltage threshold $(\geq 100 \mathrm{kV})$ allows more accurate PB assessment in CTCA.

\section{A STANDARDISED NUMERICAL FFRCT REPORTING PROPOSAL TO PRAGMATICALLY GUIDE APPROPRIATE CATHETER LABORATORY USE WITHIN A NICE COMPLIANT CHEST PAINS PATHWAY}

Matthias Schmitt. Manchester University NHS Foundation Trust, UK

\subsection{6/heartjnl-2019-BSCI.12}

Background As per NICE CG 95 11/2016 iteration Cardiac $\mathrm{CT}$ is now the first line investigation for patients with stable chest pains of assumed cardiac origin. NICE (MTG 32) also endorses the use of Heartflow FFRCT assessment for studies with intermediate lesion severity. The introduction of CACRDS and CAD-RDS reporting is facilitating a standardised reporting and provides both recommendations for appropriate initiation of preventive therapy and appropriate further assessment respectively. However, there is no general consensus on how to integrate the output of Heartflow FFRCT assessment into a coherent reporting structure.

Objective To provide, similar to CAC-RDS and CAD-RDS, a pragmatic standardised FFRCT reporting scheme which emphasises the presence of Lesion specific ischaemia (LSI) in the context of the lesion location over and above the sole numerical FFR value adjudication in order to enhance the role of Cardiac CT as a tool to appropriately enrich the catheter laboratory population and aide clinical decision making following CCTA+FFRCT.

Methods The disease spectrum of 196 consecutive FFRCT studies acquired over a 6 month period in a single UK centre was reviewed and subsequently graded according to; 1) the lowest FFRCT in any analysable vessel as negative (FFRCT $>0.8$ ), grey zone $(0.8 \geq$ FFRCT $>0.75)$, positive (FFRCT $<0.75)$. 2) The number $(\mathrm{Nr})$ of affected coronaries (1-4). 3) The presence/absence of LSI defined as localised (within a proximal or mid segment) pressure drop and subdivided in + ve $(++)$ and grey zone LSI $(+)$. 4) The location of the LSI, i.e. proximal (A) coronary segments (SCCT segments 1,5,6,11), mid (B) segments and proximal side-branches (SCCT segments $2,3,7,9,12,13,17$ ), and distal (C) segments (SCCT segments $4,8,14,15,16,18)$. Using these criteria a simple numerical scoring system was developed which may aide pragmatic clinical decision making. Exemplar illustration is provided below.

Results Out of 839 patients 196 (23.3\%) were CAD-RDS 3/ 4A and were sent for FFRCT analysis. Of those 196 patients $33.7 \%$ were FFRCT +ve (FFRCT <0.75), 20.9\% were grey zone FFR (0.75-0.799) and 45.4\% were FFRCT negative (FFRCT $=>0.8)$. One scan $(0.5 \%)$ was returned for quality reasons (image motion). LSI as defined above occurred in 50 scans $(25.5 \%)$.

Conclusion Here we present a proposal of standardised FFRCT scoring, which categorises the presence of LSI and
- -ve FFRCT $(=/>0.8)$ in all vessels

- Grey zone FFRCT (0.75-0.79)

- Nr affected vessels $1 / 2 / 3 / 4$;

- LSI present/absent; $+/-$;

- Lesion location; $\left(\mathrm{A}^{*} / \mathrm{B}^{*} / \mathrm{C}^{*}\right)$;

- NoLSI

- +ve $(<0.75)$

- $\mathrm{Nr}$ affected vessels $1 / 2 / 3 / 4$

- LSI present/absent; ++/- [FFRCT<0.75]

- LSI grey zone; +/- [FFR0.75-0.79]

- Lesion location; $\left(A^{*} / B^{*} / C^{*}\right)$

- No LSI

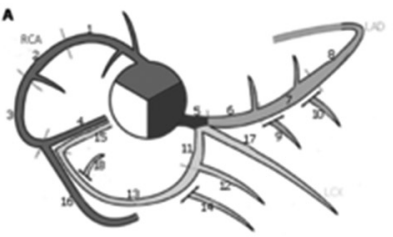

Additive Score

scores 1

2 vessels score $1,>2$ scores 2

+ in A scores 2, + in B scores 1

scores 2

2 vessels score $1,>2$ scores 2

++ in $A$ and $B$ score 2

+ in $A$ and $B$ scores 1

Score 0-2; Favours conservative

management, IA unlikely to lead to revascularisation.

Score 3; Consider stress echo/FI

Score $>\mathbf{3}$; Invasive coronary angiography

likely appropriate and increasingly high

likelihood of revascularisation

${ }^{*} \mathrm{~A}$; Proximal (segments $1,5,6,11$ ), ${ }^{*} \mathrm{~B} / \mathrm{mid} 2,3,7,9,13,17,12,{ }^{*} \mathrm{C}$ distal coronary segment $4,16,14,15,18,8$

Abstract 12 Figure 1 Examples 
Grey zone (GZ) spectrum; FFRCT (0.75-0.79), Affected vessels $1 / 2 / 3 / 4$, LSI present (A*/B*/C*), LSI absent

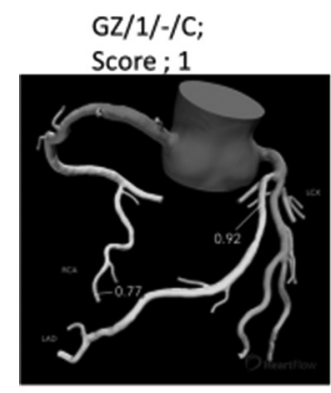

Grey zone; distal PDA of RCA "minute myocardium at risk" and no LSI, initial conservative management appears appropriate

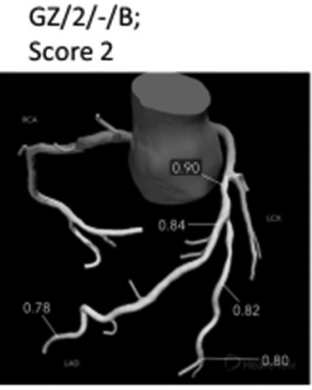

Grey zone; distal LAD and distal large diagonal, "small ischemic territory" but no LSI, conservative management again appears appropriate

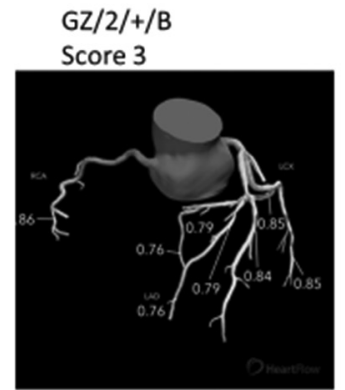

Grey zone mid LAD lesion with LSI and potentially moderate sized "ischemia / area at risk" in addition to diffuse pressure loss in $\mathrm{CX}$. Seeking further assurance in appropriate clinical context appears appropriate.
$\mathrm{GZ} / 2 /+/ \mathrm{A}$;

Score 4

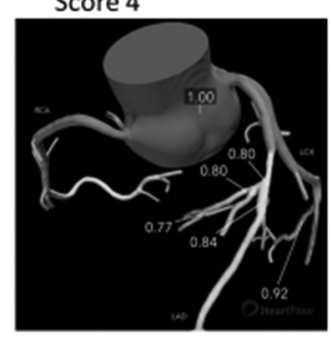

Grey zone of prox LAD involvin septal branch yet caliber jump in LAD (LSI; FFR 0.8) with ; "small ischemic territory" but potentially large "at risk territory" seeking further reassurance in appropriate clinical context appears reasonable

Abstract 12 Figure 2

\section{FFR +ve $(<0.75)$ spectrum; Affected vessels $1 / 2 / 3 / 4$, LSI present} $\left(A^{*} / B^{*} / C^{*}\right)$, LSI absent

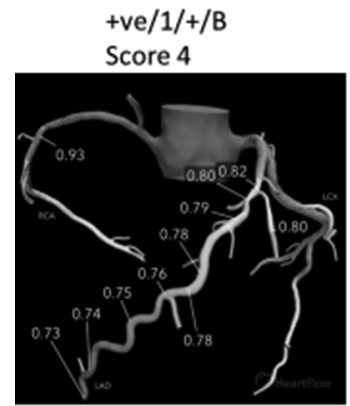

+ve study, yet only grey zone LSI in mid LAD with only distal territory +ve. Given moderate-large area subtended further clarification appears justifiable.

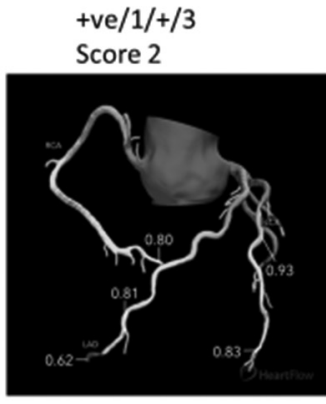

+ve yet only in very distal LAD with below $2 \mathrm{~mm}$ calibre. Risk benefit assessment probably in favour of conservative management.

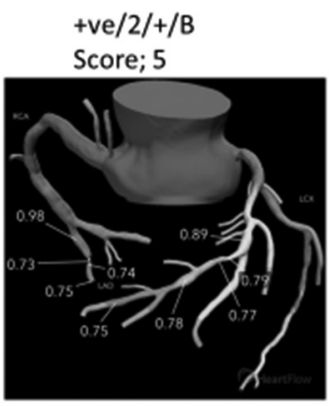

+ve (just) with grey zone LSI in mid LAD and distal RCA. If symptomatic further assessment appears reasonable.

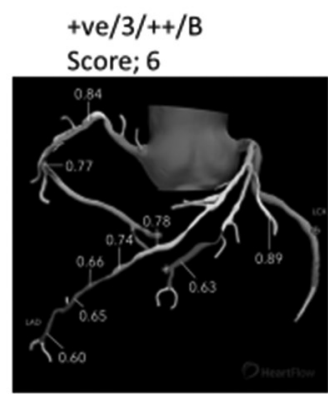

+ve with "+ve LSI" in 2 mid segments of LCA. Onward referral for IA is highly appropriate.

\section{Abstract 12 Figure 3}

lesion location in addition to the FFRCT value. If appropriately contextualised and communicated such a reporting scheme may further improve catheter laboratory utilisation and improve clinical decision making.

\section{A YEAR OF ACUTE MYOCARDITIS IN NORTHERN ALBERTA}

Emer Sonnex, Richard Coulden. University of Alberta Hospital, Edmonton, Canada

10.1136/heartjnl-2019-BSCl.13

Introduction Acute myocarditis (AM) is a major cause of troponin positive chest pain in patients without obstructive coronary disease. Many cases relate to viral infection. Drug toxicity, alcohol and auto-immune diseases have also been implicated. Diagnosis is difficult and cardiac MR (CMR) can confirm/exclude the diagnosis. We reviewed all cases of suspected AM referred for CMR in 2017.

Methods Patients were identified from referral information recorded in the CMR daybook. All cases underwent pre- \& post-contrast imaging to assess bi-ventricular function, myocardial oedema and late gadolinium enhancement (LGE).

Results Of 1753 adult patients undergoing CMR, 95 (5\%) were for suspected myocarditis. 37 had no troponin rise or peak troponin $\mathrm{I}<0.5 \mu \mathrm{g} / \mathrm{L}$ (normal range $\leq 0.15 \mu \mathrm{g} / \mathrm{L}$ ). None of these had AM by MRI criteria (15 dilated cardiomyopathy, 1 pericarditis, $1 \mathrm{LV}$ hypertrophy and 20 normal). Of the 